\title{
Performance Evaluation of Routing Protocols IN Wireless SENSOR NETWORKS
}

\author{
Laiali Almazaydeh, Eman Abdelfattah, Manal Al- Bzoor, and Amer Al- Rahayfeh \\ Computer Science and Engineering Department \\ University Of Bridgeport, Bridgeport, CT 06601, USA \\ \{lalmazay, eman, malbzoor, aalrahay\}@bridgeport.edu
}

\begin{abstract}
The efficiency of sensor networks strongly depends on the routing protocol used. In this paper, we analyze three different types of routing protocols: LEACH, PEGASIS, and VGA. Sensor networks are simulated using Sensoria simulator. Several simulations are conducted to analyze the performance of these protocols including the power consumption and overall network performance. The simulation results, using same limited sensing range value, show that PEGASIS outperforms all other protocols while LEACH has better performance than VGA. Furthermore, the paper investigates the power consumption for all protocols. On the average, VGA has the worst power consumption when the sensing range is limited, while VGA is the best when the sensing range is increased.
\end{abstract}

\section{KEYWORDS}

Sensor Networks, Routing protocol, LEACH, PEGASIS, VGA, Sensoria simulator, Energy consumption, network performance.

\section{INTRODUCTION}

A wireless sensor network is an active research area with numerous workshops and conferences arranged each year. A Wireless Sensor Networks (WSN) is a set of hundreds or thousands of micro sensor nodes that have capabilities of sensing, establishing wireless communication between each other and doing computational and processing operations [1].

Sensor networks have a wide variety of applications and systems with vastly varying requirements and characteristics. The sensor networks can be used in Military environment, Disaster management, Habitat monitoring, Medical and health care, Industrial fields, Home networks, detecting chemical, Biological, radiological, nuclear, and explosive material etc. Deployment of a sensor network in these applications can be in random fashion (e.g., dropped from an airplane) or can be Planted manually (e.g., fire alarm sensors in a facility). For example, in a disaster management application, a large number of sensors can be dropped from a helicopter. Networking these sensors can assist rescue operations by locating survivors, identifying risky areas, and making the rescue team more aware of the overall situation in the disaster area [2].

Figure 1 shows the schematic diagram of sensor node components in which sensor nodes are shown as small circles. Basically, each sensor node comprises sensing, processing, transmission, mobilizer, position finding system, and power units (some of these components are optional like the mobilizer). The same figure shows the communication architecture of a WSN. Sensor nodes are usually scattered in a sensor field, which is an area where the sensor nodes are deployed. 


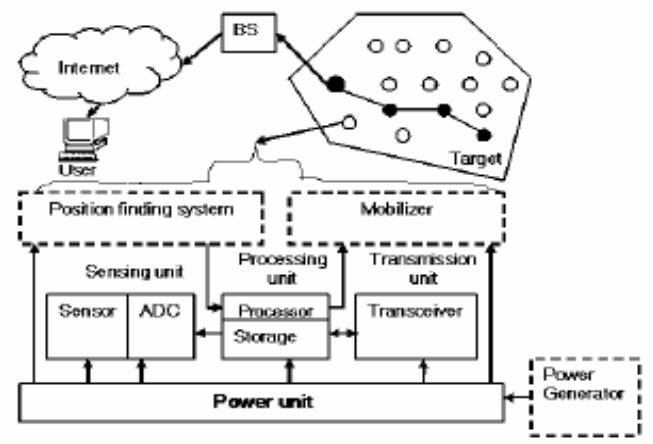

Figure 1. Structural view of sensor network [2]

Each of these scattered sensor nodes has the capability to collect and route data either to other sensors or back to an external base station(s). A base-station may be a fixed node or a mobile node capable of connecting the sensor network to an existing communications infrastructure or to the Internet where a user can have access to the reported data [2].

In general, classification of a WSN routing methodology can be done into two main categories; based on network structure or based on the protocol operation. Depending on the network structure, different routing schemes fall into this category. A sensor network can be non hierarchical or flat in the sense that every sensor has the same role and functionality. Therefore the connections between the nodes are set in short distance to establish the radio communication. Alternatively, a sensor network can be hierarchical or cluster-based hierarchical model, where the network is divided into clusters comprising of number of nodes. Cluster head, which is master node, within each respective cluster is responsible for routing the information to other cluster head. Another class of routing protocols is based on the location information of the sensor nodes either estimated on the basis of incoming signal strengths or obtained by small low-power GPS receivers or even by combination of the two previous methods. Location-based protocols use this information to reduce the latency and energy consumption of the sensor network. Our work in this paper is based on hierarchal schema to analyze the network performance.

The remainder of the paper is organized as follows: Section 2 reports related work of the wireless sensor networks protocols. In Section 3, we present the simulation model used in this paper. Section 4 provides the simulation results. Finally, Section 5 offers conclusions.

\section{RELATED WORK}

Hierarchical routing performs energy-efficient routing in WSNs, and contributes to overall system scalability and lifetime. In a hierarchical architecture, sensors organize themselves into clusters and each cluster has a cluster head, i.e. sensor nodes form clusters where the low energy nodes are used to perform the sensing in the proximity of the phenomenon. The less energyconstrained nodes play the role of cluster-heads and process, aggregate and forward the information to a potential layer of clusters among themselves toward the base station. In this section, we introduce three cluster based scheduling mechanisms.

\subsection{LEACH protocol}

Heinzelman, et.al [3] introduced a hierarchical clustering algorithm for sensor networks, called Low Energy Adaptive Cluster Hierarchy - based protocol (LEACH). In LEACH the operation is divided into rounds, during each round a different set of nodes are cluster-heads $(\mathrm{CH})$. Nodes that have been cluster heads cannot become cluster heads again for P rounds. Thereafter, each node has a $1 / \mathrm{p}$ probability of becoming a cluster head in each round. At the end of each round, 
each node that is not a cluster head selects the closest cluster head and joins that cluster to transmit data. The cluster heads aggregate and compress the data and forward it to the base station, thus it extends the lifetime of major nodes. In this algorithm, the energy consumption will distribute almost uniformly among all nodes and the non-head nodes are turning off as much as possible. LEACH assumes that all nodes are in wireless transmission range of the base station which is not the case in many sensor deployments. In each round, LEACH has cluster heads comprising $5 \%$ of total nodes. It uses Time Division Multiple Access (TDMA) as a scheduling mechanism which makes it prone to long delays when applied to large sensor networks. Figure 2 shows the communications in LEACH protocol.

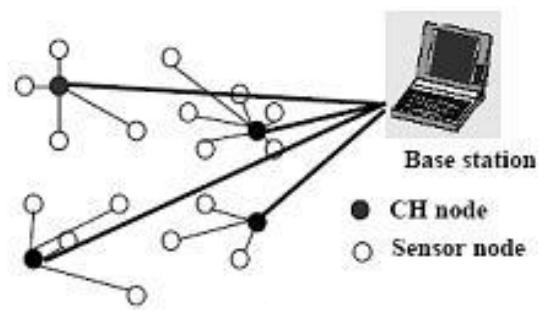

Figure 2. LEACH [7]

\subsection{PEGASIS protocol}

An enhancement over LEACH protocol was proposed in [4]. The protocol, called PowerEfficient Gathering in Sensor Information Systems (PEGASIS). The protocol is a near optimal chain-based protocol for extending the lifetime of network.

In PEGASIS, each node communicates only with the closest neighbour by adjusting its power signal to be only heard by this closest neighbour. Each Nodes uses signal strength to measure the distance to neighbourhood nodes in order to locate the closest nodes. After chain Formation PEGASIS elects a leader from the chain in terms of residual energy every round to be the one who collects data from the neighbours to be transmitted to the base station. As a result, the average energy spent by each node per round is reduced. Unlike LEACH, PEGASIS avoids cluster formation and uses only one node in a chain to transmit to the Base station instead of multiple nodes. This approach reduces the overhead and lowers the bandwidth requirements from the BS. Figure 3 shows that only one cluster head leader node forward the data to the BS.

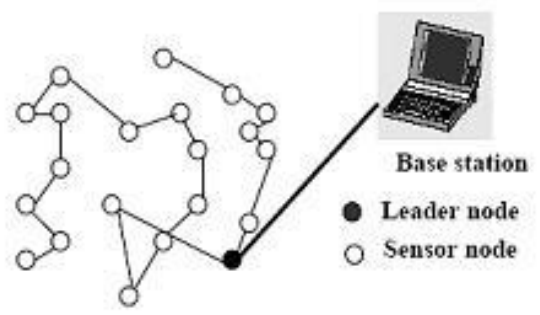

Figure 3. PEGASIS [7]

\subsection{VGA Protocol}


Virtual Grid Architecture (VGA) is an energy-efficient routing paradigm proposed in [5]. The protocol utilizes data aggregation and in-network processing to maximize the network lifetime. Due to the node stationary and extremely low mobility in many applications in WSNs, a reasonable approach is to arrange nodes in a fixed topology. A GPS-free approach is used to build clusters that are fixed, equal, adjacent, and non-overlapping with symmetric shapes. In [5], square clusters were used to obtain a fixed rectilinear virtual topology. Inside each zone, a node is optimally selected to act as $\mathrm{CH}$. Data aggregation is performed at two levels: local and then global. The set of CHs, also called Local Aggregators (LAs), perform local aggregation, while a subset of these LAs are used to perform global aggregation. However, the determination of an optimal selection of global aggregation points, called Master Aggregators (MAs), is NP-hard. Figure 4 illustrates an example of fixed zoning and the resulting virtual grid architecture (VGA) used to perform two level data aggregation. Note that the location of the base station is not necessarily at the extreme corner of the grid; rather it can be located at any arbitrary place.

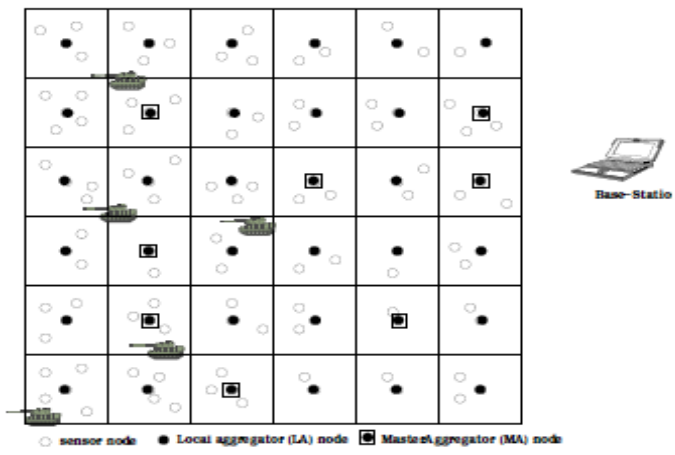

Figure 4. Regular shape tessellation applied to the network area [7]

Table 1 shows a comparison between LEACH, PEGASIS, and VGA routing protocols according to their design characteristics [8].

Table1. Classification and Comparison of routing protocols in WSNs [8]

\begin{tabular}{|l|l|l|l|l|l|l|l|l|}
\hline $\begin{array}{c}\text { Routing } \\
\text { Protocols }\end{array}$ & Classification & $\begin{array}{c}\text { Power } \\
\text { Usage }\end{array}$ & $\begin{array}{c}\text { Data } \\
\text { Aggregation }\end{array}$ & Scalability & $\begin{array}{c}\text { Query } \\
\text { Based }\end{array}$ & $\begin{array}{c}\text { Over } \\
\text { head }\end{array}$ & $\begin{array}{c}\text { Data } \\
\text { delivery } \\
\text { model }\end{array}$ & QoS \\
\hline LEACH & $\begin{array}{l}\text { Hierarchical / } \\
\text { Node-centric }\end{array}$ & High & Yes & Good & No & High & $\begin{array}{l}\text { Cluster- } \\
\text { head }\end{array}$ & No \\
\hline PEGASIS & Hierarchical & Max & No & Good & No & Low & $\begin{array}{l}\text { Chains } \\
\text { based }\end{array}$ & No \\
\hline VGA & Hierarchical & Low & Yes & Good & No & High & Good & No \\
\hline
\end{tabular}

\section{SimULATION ENVIRONMENT}

In this section we present the simulation results of LEACH, PEGASIS, and VGA followed by a comparison among the different techniques.

\subsection{Simulation Platform}

Sensoria simulator for simulating different routing protocols is used as experiment platform. Sensoria is a fully fledged simulator for WSNs. It is a very powerful in simulating a range of 
small to large scale WSNs based on a simple and complete Graphical User Interface (GUI). Sensoria's GUI allows users to design various simulation scenarios and display the simulation results graphically with many formats [6].

\subsection{Experiment Environment}

In this section we define the common parameters that are used in all simulation scenarios.

\subsubsection{Network Specifications}

To evaluate the performance of the hierarchal routing protocols, the simulation consists of 80 homogeneous nodes with initial energy of 0.5 Joule, scattered randomly within a $40 \times 40 \mathrm{~m}$ sensor field (see Figure 5). The Base Station is (BS) located at $(25,150) \mathrm{m}$, so it is at least $110 \mathrm{~m}$ far from the closest packets and 248 bit control packets. The energy consumption due to communication will be calculated using the first order energy model. We assume that each sensor node generates one data packet per time unit to be transmitted to the BS. For simplicity, we refer to each time unit as a round. During the simulation process, only a set of sources will be selected randomly at each round to send their data to the BS. Transmission and sensing range are $15 \mathrm{~m}$ and $8 \mathrm{~m}$ respectively.

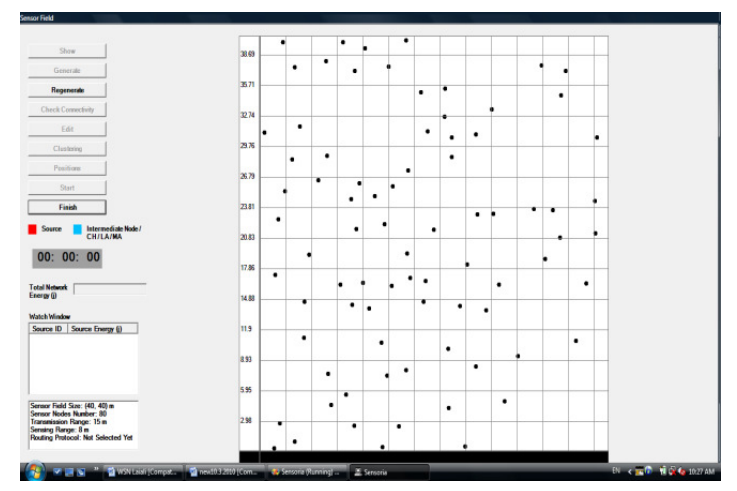

Figure 5. Simulated WSN with node distribution, $n=80$

\subsubsection{Network Protocols}

\section{Physical parameters}

Physical parameters are the same of all three protocols as follows

- Transmission speed $100 \mathrm{bit} / \mathrm{s}$

- Network bandwidth 5000 bit/s

- Data packet processing delay $0.1 \mathrm{~ms}$

\section{Protocol parameters}

\section{LEACH parameters:}

Cluster type Dynamic

CHs percentage (\%) 5.0

$\mathrm{CHs}$ selection cycle $1.00 \mathrm{~s}$

\section{PEGASIS parameters:}

No special parameters for PEGASIS 
VGA parameters:

LAs and MAs selection cycle $1.00 \mathrm{~s}$

\subsubsection{Simulation parameters}

Required task: we configure the simulation process to give a Periodic sensing every 2 second.

\subsubsection{Simulation criteria}

Two scenarios are used to measure the performance, as follows:

A) When the value of transmission range is $15 \mathrm{~m}$

B) When the value of transmission range is $70 \mathrm{~m}$

These two scenarios are used according to the Loss of network connectivity. Sensoria will report the network life time at the round when a sensor node is isolated (all its neighbours run out of energy), i.e. the network is not fully connected.

\section{RESULTS AND DISCUSSION}

In the first scenario we ran the simulations to determine the number of rounds of communication when the network loss its connectivity using LEACH, PEGASIS, and VGA with each node having the same initial energy level and transmission range which are 0.5 Joule and $15 \mathrm{~m}$ respectively. Our simulations show the following results:

1. The lifetimes of the network are 2174, 1017, and 28 rounds for PEGASIS, LEACH, and VGA respectively, Figure 6 shows the network life time.

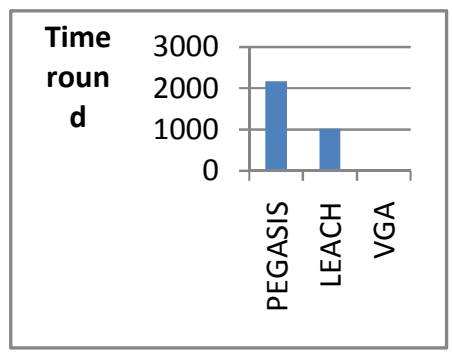

Figure 6. Network life time

2. The latest total network energy is $0.1170,4.4354$ and 13.2178 for PEGASIS, LEACH and VGA. The minimum value is achieved by PEGASIS, since it has loss connectivity after the round time for LEACH and PEGASIS. Figure 7, 8 and 9 respectively shows the total network energy versus time for the three routing algorithms.

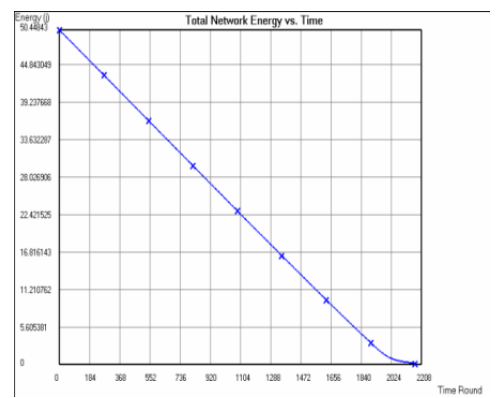


Figure 7. PEGASIS total network energy

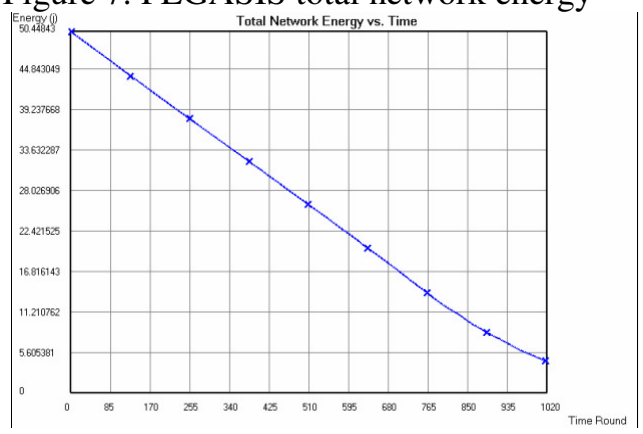

Figure 8. LEACH total network energy

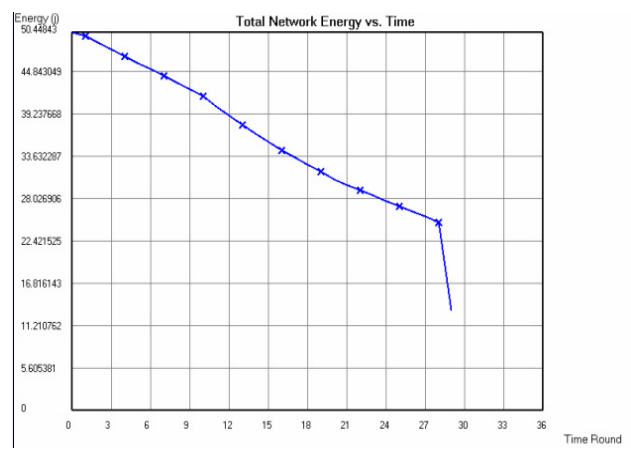

Figure 9. VGA total network energy

3. The number of failed nodes became 99 at the last period in PEGASIS, 53 in LEACH and 49 in VGA Figure 10,11 and 12 respectively shows the number of failed nodes versus time for the three routing algorithms.

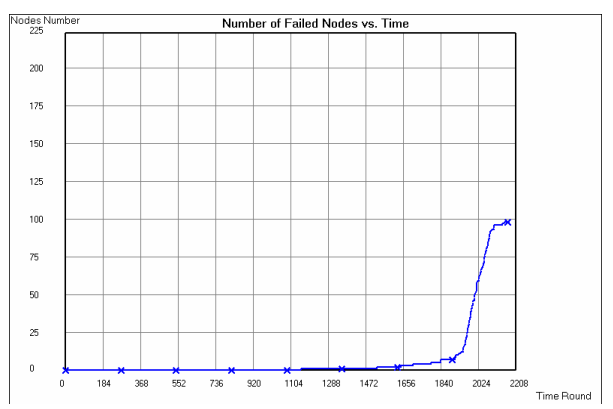

Figure 10. PEGASIS failed nodes

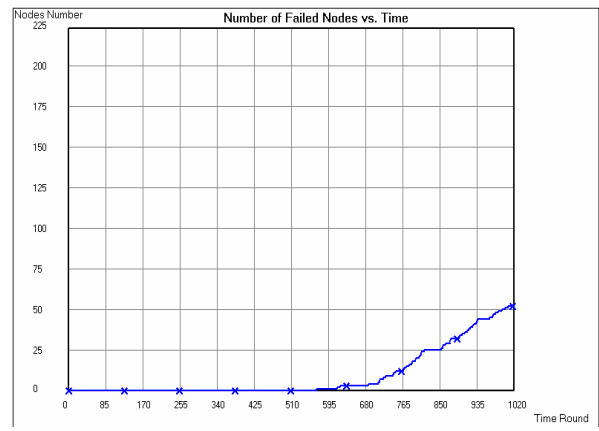


Figure 11. LEACH failed nodes

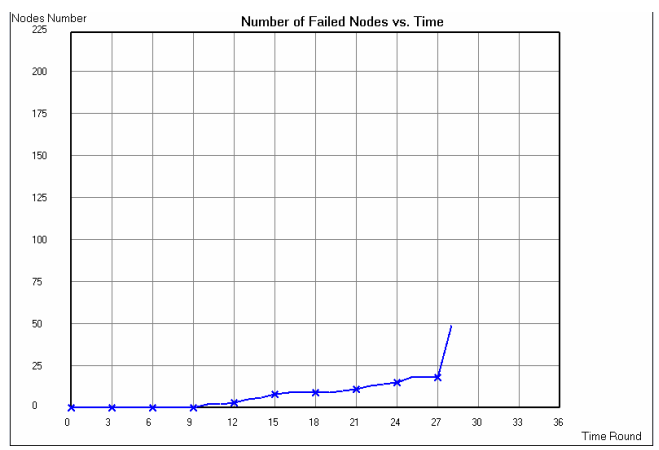

Figure 12. VGA failed nodes

We conclude that PEGASIS achieves approximately $2 \mathrm{x}$ the number of rounds compared to LEACH. It approximately achieves $77 \mathrm{x}$ the number of rounds compared to VGA. LEACH approximately performs $36 \mathrm{x}$ the number of rounds compared to VGA.

We run the simulations to determine the number of rounds of communication when the network loss its connectivity, where this time the transmission range is $70 \mathrm{~m}$. Figure 13,14 respectively shows the clustering in VGA when the transmission range is $15 \mathrm{~m}$ and when the transmission range is $70 \mathrm{~m}$.

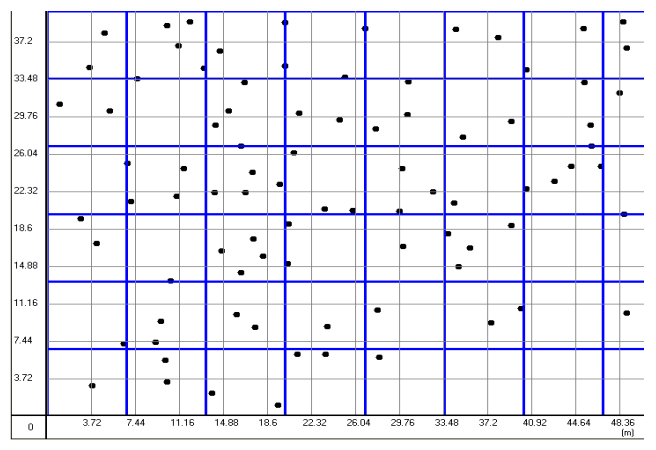

Figure 13. Clustering in VGA when transmission range is $15 \mathrm{~m}$

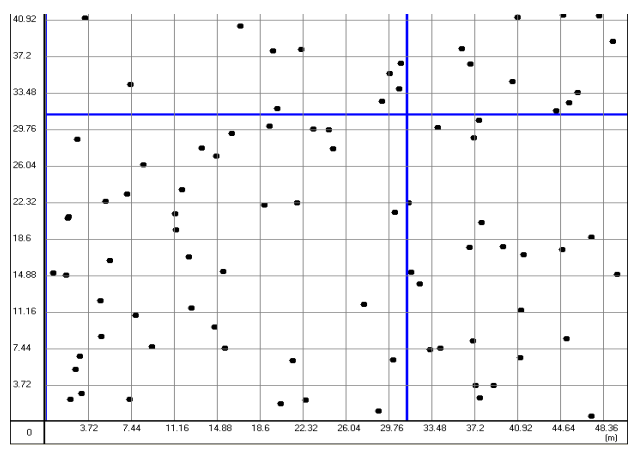


Figure 14. Clustering in VGA when transmission range is $70 \mathrm{~m}$

Our simulations show that VGA approximately achieves $3 \mathrm{x}$ the number of rounds compared to PEGASIS. It approximately achieves $11 \mathrm{x}$ the number of rounds compared to LEACH. While PEGASIS approximately performs $3 \mathrm{x}$ the number of rounds compared to LEACH. The lifetimes of the network are 8124, 2285, and 741 rounds for VGA, PEGASIS, and LEACH respectively. Figure 15 shows the network lifetime.

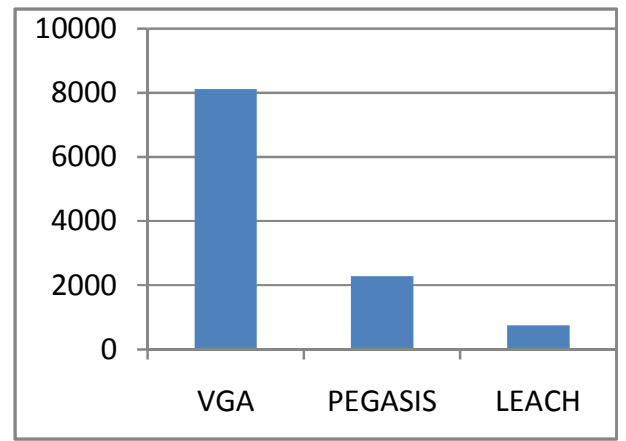

Figure15. Network lifetime

\section{CONCLUSIONS}

In this paper we presented a simulation model for LEACH, PEGASIS, and VGA hierarchical routing protocols. Sensoria simulator is used to compare the performance of the three routing protocols. The simulation results show that PEGASIS can greatly prolong sensor network's lifetime when the transmission range is limited. VGA saves more energy than other protocols when the transmission range is farther, since the early death of the nodes reduces the network's coverage badly.

\section{ACKNOWLEDGEMENTS}

We thank Dr. Jamal N. Al-karaki and Ghada A. Al-Mashaqba to provide us with the Sensoria simulator, open source version.

\section{REFERENCES}

[1] K. Khamforoosh, and H., Khamforoush, "A new rounting Algorithm for Energy Reduction in Wireless Sensor Networks", IEEE, 2009

[2] J.N Al-Karaki, and A.E. Kamal, "Routing techniques in wireless sensor networks: a survey", IEEE Wireless Communications, Vol. 11, No. 6, pp.6-28, December 2004.

[3] M. Younis, M. Youssef and K. Arisha, "Energy-Aware Routing in Cluster-Based Sensor Networks", in the Proceedings of the 10th IEEE/ACM(MASCOTS2002), Fort Worth, TX, October 2002

[4] S. Lindsay and C. Raghavendra, "PEGASIS: Power-Efficient Gathering in Sensor Information Systems", international Conf. on Communications, 2001. Anew routing

[5] J.N Al-karaki et al.,'Data Agggregation in Wireless Sensor Networks - Exact and Approximate Algorithms," Proc. IEEE Wks. High Perf. Switching and Routing 2004, Phoenix, AZ, Apr. 18-21, 2004.

[6] J. N. Al-Karaki, and G. A. Al-Mashaqbeh, "SENSORIA: A New Simulation Platform for Wireless Sensor Networks", International Conference on Sensor Technologies and Applications, 2007.

[7] http://alkautsarpens.wordpress.com/wsn/

[8] R. V. Biradar, V. C. Patil, Dr. S. Sawant, and Dr. R. R. Mudholkar, "Classification and comparisson of routing protocols in wireless sensor networks", UbiCC Journal, Vol. 4. 


\section{Authors}

Laiali Almazaydeh is a Ph.D. student of Computer Science and Engineering at the University of Bridgeport. She received a B.S. in Computer Science from Al-Hussein Bin Talal University and an M.S. in Computer Information Systems from The Arab Academy for Banking and Financial in 2003 and 2007, respectively. Her current research focuses on the wireless sensor networks and human computer interaction.

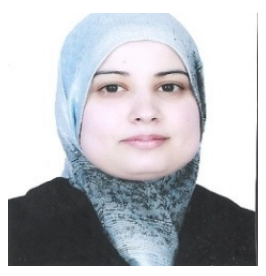

Eman Abdelfattah had received the MS Degree in Computer Science from the University of Bridgeport in 2002. Currently, she is working as an adjunct instructor at the University of Bridgeport. She has research interests in the areas of networking and communications. Her research results were published in prestigious international conferences in circuits and VLSI design. She actively participated as a committee member of the International Conferences on Engineering Education, Instructional Technology, Assessment, and E-learning EIAE 05, EIAE 06, EIAE 07, EIAE 08, and EIAE 09.

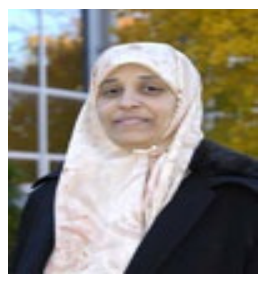

Manal AL-Bzoor is a Ph.D. student in Computer Science and Engineering at University of Connecticut. She has received her B.S. degree from Jordan University of Science and Technology Jordan and her MS degree in Computer Engineering from University of MichiganDearborn in 2004. She was awarded two scholarships from Yarmouk University for Masters Study and for current PhD study. Manal current research interests are in wireless sensor networks and distributed/parallel systems.

Amer Al-Rahayfeh is a Ph.D. student of Computer Science and Engineering at the University of Bridgeport. He received a B.S. in Computer Science from Mutah University and an M.S.in Computer Information Systems from The Arab Academy for Banking and Financial in 2002 and 2004. He worked as an Instructor at The Arab Academy for Banking and Financial (2006-2008), Amer current research interests are in multimedia database systems.
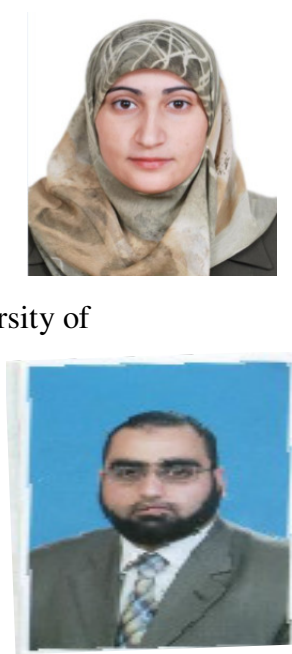